\title{
Behavioral factors affecting soil-transmitted helminthiasis among pregnant women attending antenatal care follow-up in Dembia, Ethiopia
}

\section{Teshager Assefa}

Dembia health office

\section{Lemma Derseh}

University of Gondar College of Medicine and Health Sciences

Moges Muluneh Boke ( $\sim$ Mogelove75@gmail.com )

University of Gondar College of Medicine and Health Sciences https://orcid.org/0000-0001-7940-2691

Temesgen Yihunie Akalu

University of Gondar College of Medicine and Health Sciences

\section{Research}

Keywords: West Dembia, soil-transmitted helminthiasis, pregnant women

Posted Date: July 28th, 2021

DOI: https://doi.org/10.21203/rs.3.rs-746479/v1

License: @ (i) This work is licensed under a Creative Commons Attribution 4.0 International License.

Read Full License 


\section{Abstract \\ Background}

Globally, 2 billion people infected with soil-transmitted helminthiasis, nearly 250 million women live in helminthiasis endemic area. Sub-Saharan Africa is the most affected region by soil-transmitted helminths. The prevalence of soil-transmitted helminthiasis and its determinants among pregnant mothers was not well known in this study area. Therefore, the current study was aimed to determine the prevalence of soil-transmitted infestation and its determinants in the West Dembia district.

\section{Methods}

A facility-based cross-sectional study design was used from October to November 2020 in the West Dembia district. A systematic random sampling technique was used to take part 416 pregnant women. Data were collected using a pretested standardized questionnaire and stool sample was collected from each participant. Data were entered into Epi-info version 7 and exported to Stata version 14 for analysis. A crude Odds Ratio (COR) and an Adjusted Odds Ratio (AOR) with a 95\% Confidence Interval (Cl) were computed. The goodness of fit for the model was checked using Hosmer and Lemeshow test.

\section{Results}

Of all, 416 pregnant women were examined and 115 (27.6\%) of women were positive at least to one of the common soil-transmitted helminthiases. Out of the total cases, Ascaris, Hookworm, and Schistosoma species account for $45(39 \%), 44(38 \%)$, and $24(21 \%)$, respectively. Rural dwellers AOR: $1.9,(95 \%$ Cl: 1.04 , 3.67), never using latrine AOR: 3.6, (95\% Cl: 1.35, 9.74), not using soap for handwashing AOR: 2.7: (95\% Cl: $1.31,5.36)$, eating raw vegetables AOR: $2.4,(95 \% \mathrm{Cl}: 1.44,3.94)$, the habit of eating soil AOR: 3.4 , (95\% Cl: $1.22,9.43)$ were statistically significantly associated with soil-transmitted helminthiasis infestation.

\section{Conclusion}

The prevalence of soil-transmitted helminthiasis infestation among pregnant women is high. Rural dwellers, not using a latrine, not using soap for handwashing, eating raw vegetables, and habit of eating soil were predictors to the infestation of soil-transmitted helminthiasis among pregnant women. Therefore, besides strengthening chemotherapy prevention, Antenatal care health promotion activities need to focus on behavioral change.

\section{Introduction}

Soil-transmitted helminthiasis (STH) is a term that refers to a group of parasitic diseases caused by worms that are transmitted to humans by feces-contaminated soil (2). Soil-transmitted helminthiasis 
once expelled from the feces of the infected individual requires a period of maturation in the soil before becoming infective (3).

Soil-transmitted helminths (STH) infestation is widely distributed in many countries across the globe. Nearly a quarter of the global population is infected with soil-transmitted helminths. Around 250 million girls and adults' women live in the soil-transmitted helminthiasis endemic areas in the world. Globally, more than 100 countries were found in soil-transmitted helminths endemic areas. Sub-Saharan Africa, the Americas, and Asia countries share the largest number (4). In Ethiopia, an estimated 81 million people live in STH endemic areas (5)(6). A segment study conducted in Ethiopia showed that soil-transmitted helminthiasis is still a great public health problem in some regions of the country. According to a recent three-series Ethiopian ministry of health report, soil-transmitted helminth placed in the top ten morbidity causes disease list (6). The prevalence varies from place to place (7). In the Amhara region of Ethiopia, in earlier surveys report, seven in ten pregnant women infected with soil-transmitted helminth (7). Poor sanitation and low clean water coverage is a most common risk factor to soil-transmitted helminthiasis (8).

Pregnant women are among the second-high risk population groups next to children for soil-transmitted helminth (9). Even if both pregnant women and children were the risky group to soil-transmitted helminth infestations. However, the previous researchers mainly focused on only preschool and school-age children. The studies on the burden of soil-transmitted helminthiasis among pregnant women is limited. Therefore, the current study is aimed to determine the prevalence of soil-transmitted helminth and its associated factors among pregnant women in the West Dembia district.

\section{Material And Methods}

\section{Study area, design, and period}

A facility-based cross-sectional study design was conducted from October to November 2020 in West Dembia district, northwest Ethiopia. The district is found in the central Gondar zone 55 kilometers $(\mathrm{km})$ far from Gondar town, which is the capital of the central Gondar zone, a total population of the district is 132,671 . Among these 65,501 (49.4\%) they were females and 4,471 (3.7\%) of the females are expected to be pregnant. Regarding health facility coverage, the district has 5 health centers and 20 health posts that render ANC services for pregnant women. A total of 203 health professionals in different disciplines providing service and of these $16(8 \%)$ of them were midwives and $9(4.4 \%)$ of them were laboratory professionals. All pregnant women who were attending antenatal clinics at health institutions of the district were eligible for the study.

\section{Sample size determination and sampling procedures}

The sample size was determined using double population proportion with assumptions of $95 \%$ confidence interval, $80 \%$ power, and percent of outcome from previous study $43.8 \%$ and 0.05 level of significance. Through this assumption, the sample size was calculated using EPI-info version 7 software 
and a total of 416 sampled pregnant women were included in the study. First, we proportionally allocated the estimated sample size to each health facilities (five health centers) based on their number of pregnant women attended ANC, then final to recruit the study participants systematic random sampling was applied. Pregnant women who were attending their ANC in health centers were recruited regardless of their trimester.

\section{Data collection tools and procedures}

Orientation was given on how to collect stool specimens and provide them with a leak-proof plastic container. Data were collected by structured questionnaires using the interviewer-administered technique. A single stool specimen of about 5 grams (thumb size) was collected from each study participant using a clean, dry, and leak-proof plastic container labeled with a unique identification number. A portion of the sample was processed by formol ether concentration technique, $7 \mathrm{ml}$ formol water was added into a 1gram (pea-sized stool) sample, mixed with a wooden stick then $3 \mathrm{ml}$ ether were added, mix well. The stool sample was well mixed and filtered using a funnel with gauze and centrifuged for 1 minute at 3000 revolutions per minute. Finally, the supernatant was discarded, and the sediment was examined by laboratory professionals (10).

\section{Data quality assurance mechanisms}

The questionnaire was prepared in English language and translated to Amharic language (local language of the study area). The Amharic version questionnaire was used to collect the data. The training was given to midwives and laboratory technicians. The collection procedure was supervised by the principal investigator closely. The completeness of data was checked by the supervisors and the principal investigators daily

\section{Data entry and analysis}

Data were entered into epi-info version 7 and analyzed using Stata version 14. Data cleaning, editing, and coding were made. Descriptive statistics were conducted to show frequencies and numbers. Binary logistic analysis was done to check whether there was an association between dependent and independent variables and those variables which were p-value less than 0.2 in bivariate logistic regression were entered into multivariable logistic regression. An odds ratio was used to determine the association and strength between the dependent and independent variables and variables with a P-value less than 0.05 were used to declare statistical significance. Model fitness was done using Hosmer and Lemeshow test.

\section{Results}

\section{Socio-demographic characteristics of study participants}

A total of 416 pregnant women were included in the study with a response rate of $99 \%$. The mean age of the respondents was 27 years with a standard deviation (SD) of 5.8 years. The majority, $411(98.8 \%)$ of 
the pregnant women were married and 406 (97.6\%) orthodox religious followers. Of all, 355(85.3\%) of respondents were housewives. Regarding the educational status, $243(58.4 \%)$ of them were unable to read and write. Of all, $179(43 \%)$ of respondents were in the second trimester (Table 1). 
Table 1

Socio-demographic and clinical factors of pregnant women examined for STH in West Dembia district, Central Gondar, Amhara, Ethiopia, 2020

\begin{tabular}{|lll|}
\hline Variables & Frequency & Percentage (\%) \\
\hline Age of respondents & 8 & 1.9 \\
$<18$ years & 126 & 30.3 \\
18-24 years & 230 & 55.3 \\
25-34 years & 52 & 12.5 \\
35-45years & & \\
\hline Residence & 103 & 24.76 \\
Urban & 313 & 75.24 \\
Rural & & \\
\hline Educational status of respondents & 243 & 58.4 \\
Unable to read and write & 61 & 14.7 \\
Able to read and write & 46 & 11.1 \\
Elementary school complete & 35 & 8.4 \\
High school complete & 31 & 7.5 \\
Certificate and above & & \\
\hline Occupational status of respondents & 354 & 85.3 \\
Housewife & 27 & 6.5 \\
Merchant & 21 & 5 \\
Government employed & 5 & 1.2 \\
Private employed & 5 & 0.7 \\
Daily laborer & 30 \\
Student & 179 \\
\hline Trimester of pregnancy & & \\
First trimester & & \\
Second trimester & & \\
Third trimester & & \\
\hline
\end{tabular}




\begin{tabular}{|llll|}
\hline Variables & Frequency & Percentage (\%) \\
\hline Gravida & 109 & 26 \\
Primigravid & 307 & 74 \\
Multigravida & & \\
Marital status & 1 & 0.2 \\
Single & 411 & 98.8 \\
Married & 4 & 1 \\
Divorced & & \\
\hline
\end{tabular}

\section{Environmental and behavioral factors}

The majority of the households, 332(79.8\%) were used water from protected sources. From total protected source water users 96 (23\%), 153(36.8\%), and 83(20\%) were used from pipe water, protected well, and protected spring, respectively. Slightly above third $(76 \%)$ of respondents had latrine and only $28.4 \%$ of them had handwashing facilities. Among the total households, $71 \%$ of them cooked foods outside the house. Regarding waste disposal experience, nearly one-third (31\%) of the households used burning to dispose of waste, $29 \%$ were used waste pits, and $40 \%$ openly dispose of the house wastes. Regarding shoes wearing experience, $72 \%$ of the total respondents wear shoes always.

\section{Prevalence of soil-transmitted helminthiasis}

The overall prevalence of soil-transmitted helminthiasis infestation among pregnant women in the West Dembia district was $27.6 \%$ (95\% Cl: 23.4, 32.2) Fig. 1. From a total soil-transmitted helminthiasis infestation, 45(39\%), 44(38\%), 24(21\%) of respondents had Ascaris, Hookworm, and Schistosoma specious, respectively.

\section{Factors associated with soil-transmitted helminthiasis}

In the bivariable logistic regression analysis residence, water from the container, latrine/toilet availability, latrine utilization, hand washing facility, child waste disposal, washing hands after toilet, using soap for handwashing, the trend of handwashing after any procedure, eating raw vegetable and a habit of soil eating were significant associated with soil-transmitted helminthiasis infestation at a p-value of less than 0.2 .

In multiple logistic regression residences, latrine utilization, using soap for handwashing, eating raw vegetables, and a habit of soil eating remained as predictors of soil-transmitted helminthiasis infestation among pregnant women.

According to the result of this study, the odds of getting soil-transmitted helminthiasis infestation were 1.9 times high in rural dwellers (AOR: 1.9, 95\% $\mathrm{Cl} 1.04,3.66$ ). The odds of getting soil-transmitted helminthiasis were 3.6 times more likely risky among pregnant women who never use latrine (AOR: 3.6, 
$95 \% \mathrm{Cl}: 1.35,9.74)$ and 2.3 times more likely risk among pregnant women who use latrine sometimes (AOR: $2.3,95 \% \mathrm{Cl}: 1.08,4.99)$. Similarly, the odds of getting STH infection were 2.7 times higher in pregnant women who do not use soap for handwashing (AOR: $2.7,95 \% \mathrm{Cl}: 1.31,5.36$ ) as compared to those pregnant women who use soap for handwashing.

Moreover, soil-transmitted helminthiasis infestation (STH) was significantly associated with eating raw vegetables, the odds of STH infection were 2.4 times greater among pregnant women who eat raw vegetables (AOR: $2.4,95 \% \mathrm{Cl}: 1.44,3.94)$ and the odds of getting STH infection were 3.4 times higher among pregnant women who have a habit of eating soil (AOR: $3.4,95 \% \mathrm{Cl}: 1.22,9.43$ ) than those pregnant women who do not have the habit of eating soil (Table 2). 
Table 2

Results of logistic regression on factors associated with STH among pregnant women in West Dembia, Central Gondar, Amhara, Ethiopia, 2020

\begin{tabular}{|c|c|c|c|c|}
\hline \multirow[t]{2}{*}{ Variables } & \multicolumn{2}{|c|}{ STH } & \multirow[t]{2}{*}{ COR $(95 \% \mathrm{Cl})$} & \multirow[t]{2}{*}{ AOR $(95 \% \mathrm{Cl})$} \\
\hline & Yes & No & & \\
\hline \multicolumn{5}{|l|}{ Residence } \\
\hline Rural & 98 & 215 & $2.3(1.30,4.09)$ & $1.9(1.04,3.66)$ \\
\hline Urban & 17 & 86 & 1 & \\
\hline \multicolumn{5}{|c|}{ Pour water from the container } \\
\hline Immersing can & 33 & 54 & $1.8(1.12,3.03)$ & $1.6(0.91,2.93)$ \\
\hline Inclining & 82 & 247 & 1 & \\
\hline \multicolumn{5}{|c|}{ latrine/toilet availability } \\
\hline No & 40 & 62 & $2.1(1.28,3.30)$ & $1.3(0.59,3.03)$ \\
\hline Yes & 75 & 239 & 1 & \\
\hline \multicolumn{5}{|c|}{ Latrine utilization } \\
\hline Never use & 10 & 32 & $3.6(1.78,7.51)$ & $3.6(1.35,9.74)$ \\
\hline Some times & 51 & 141 & $2.3(1.11,4.63)$ & $2.3(1.08,4.99)$ \\
\hline Always & 11 & 69 & 1 & \\
\hline \multicolumn{5}{|c|}{ Hand washing facility } \\
\hline No & 95 & 203 & $2.3(1.34,3.93)$ & $1.3(0.67,2.46)$ \\
\hline Yes & 20 & 98 & 1 & \\
\hline \multicolumn{5}{|c|}{ Child waste disposal } \\
\hline outside toilet & 50 & 102 & $2.0(1.24,3.15)$ & $1.4(0.79,2.46)$ \\
\hline Toilet & 32 & 130 & 1 & \\
\hline \multicolumn{5}{|c|}{ Wash hands after toilet } \\
\hline No & 67 & 109 & $2.5(1.58,3.81)$ & $1.3(0.72,2.47)$ \\
\hline Yes & 48 & 192 & 1 & \\
\hline \multicolumn{5}{|c|}{ Using soap for handwashing } \\
\hline No & 25 & 43 & $3.5(2.10,5.69)$ & $2.7(1.31,5.36)$ \\
\hline
\end{tabular}

STH: soil-transmitted helminthiasis; COR: crude odds ratio; AOR: adjusted odds ratio 


\begin{tabular}{|c|c|c|c|c|}
\hline \multirow[t]{2}{*}{ Variables } & \multicolumn{2}{|c|}{ STH } & \multirow[t]{2}{*}{$\operatorname{COR}(95 \% \mathrm{Cl})$} & \multirow[t]{2}{*}{ AOR $(95 \% \mathrm{Cl})$} \\
\hline & Yes & No & & \\
\hline Yes & 25 & 147 & 1 & \\
\hline \multicolumn{5}{|c|}{ The trend of handwashing after any procedure } \\
\hline No & 95 & 206 & $2.2(1.28,3.76)$ & $1.1(0.57,2.21)$ \\
\hline Yes & 20 & 95 & 1 & \\
\hline \multicolumn{5}{|c|}{ Eating raw vegetable } \\
\hline Yes & 57 & 91 & $2.3(1.46,3.52)$ & $2.4(1.44,3.94)$ \\
\hline No & 58 & 210 & 1 & \\
\hline \multicolumn{5}{|c|}{ A habit of Soil eating } \\
\hline Yes & 16 & 8 & $6.0(2.48,14.40)$ & $3.4(1.22,9.43)$ \\
\hline No & 98 & 294 & 1 & \\
\hline
\end{tabular}

\section{Discussion}

This study focused on the prevalence and determinants of soil-transmitted helminthiasis (STH) infestation among pregnant women attending ANC follow-up in the West Dembia district. Nearly onefourth of the study subjects had STH in the Dembia district. According to the Ministry of Health $(\mathrm{MoH})$ STH endemic area classification, there are three categories including high transmission areas (prevalence rate $>50 \%$ ), moderate transmission areas (prevalence rate in between $20 \%-50 \%$ ), and low transmission areas (less than $20 \%$ prevalence). According to WHO recommendation, moderate transmission areas of soil-transmitted helminthiasis need annual treatment (11).

This finding is comparable with the global soil-transmitted helminthiasis (STH) prevalence (24\%) report (12), studies finding obtained from Hosanna town (29.5\%) (13), Felegehiwot Hospital (31.5\%) (14), and Wollega district (24.7\%)(15). However, the current finding is lower than studies conducted in Mecha district (70.6\%) (16), and Maytsebri primary hospital (51\%) (17).

The reason for this discrepancy might be attributed to hygiene sanitation activities and latrine coverage variation. Latrine coverage and handwashing with soap in Maytsebri was $50.9 \%$ and $22.3 \%$ respectively, whereas in the current study the coverage of latrine and handwashing with soap was $75.5 \%$ and $41.3 \%$ respectively. The current study finding is also lower than the studies result obtained from Bogota, Colombia (41\%) (18), Lalo Kile, Oromia region (43.8\%) (19), and West Gojjam, Amhara region (37.3\%) (20). The reason for this discrepancy might be due to differences in environmental and socioeconomic characteristics of study participants. This finding is higher than studies obtained from Logos, Nigeria 
(8.3\%) (21), Kilifi hospital, Kenya (16.75\%) (22). The reason for this discrepancy might be the variation of latrine and proper handwashing practice coverage, which was $87 \%$ and $67 \%$ respectively in study Kenya, $100 \%$ and $92 \%$ in the study obtained from Nigeria, whereas $75.5 \%$ and $41 \%$ in the current study.

Residence, latrine utilization, using soap to hand washing, eating raw vegetables, and habit of soil eating were significantly associated with soil-transmitted helminthiasis infestation.

Being a rural resident was associated with increased odds of developing soil-transmitted helminthiasis infestation. This finding agrees with studies conducted in Mecha district (16), East Wollega, Oromia region (15), and West Gojam zone (20). This might be pregnant women in rural areas who always work on contaminated soil barefoot which is risky to STH infestation. Moreover, pregnant women living in rural areas had poor personal and environmental sanitation practices, low educational status, and lack of awareness as a result, the possibility of being infected by intestinal parasitic infection is high.

Latrine usage was significant associated with soil-transmitted helminthiasis in pregnant women. Pregnant women who had sometimes and never used latrine were more likely at risk to soil-transmitted helminthiasis infestation when compared to always latrine used. This finding in line with studies conducted in Kenya (22), West Gojam (20), and Wollega (15). That latrine utilization prevents the contamination of the soil with human faces which contain infective eggs or larvae.

The report of this study also revealed that using soap for handwashing is another determinant factor to soil-transmitted helminthiasis, women who did not use soap for handwashing were more likely to be infected, this result is consistent with studies done in Kenya (22), Maytsebri district (17), Mecha (16), and Lalo kilie (19). That proper handwashing practice breaks the chain of transmission for intestinal parasites.

The odds of soil-transmitted helminthiasis infestation were higher among pregnant women who had a habit of eating raw vegetables. This finding is comparable to the studies conducted in Kenya (22), Wollega (15), West Gojam, Amhara region (20), and Mecha district (16). This may be due to that raw vegetable acts as a vehicle for transporting the infective parasites. Similarly, the odds of infection were higher among pregnant women who have a habit of eating soil. This result is consistent with the studies conducted in Maytsebri district (17), Tigray region, and west Gojam zone, Amhara region (20). That the fecal contaminated soil may enter into the body and cause the disease (23).

Over $50 \%$ of pregnant women in low- and middle-income countries suffer from anemia, and helminthiasis is a major contributory cause in endemic areas. Soil-transmitted helminths cause iron deficiency anemia by feeding blood and reduce the absorption of iron and other nutrients by causing anorexia, vomiting, and diarrhea.

A single stool specimen was used to assess soil-transmitted helminthiasis infestation status which may underestimate the prevalence rate. The participant was only those pregnant women who came to health facilities to utilize the ANC service, this makes difficult for the generalization of the study findings. 


\section{Conclusion}

The prevalence of soil-transmitted helminthiasis among pregnant women was moderate. Residence, frequency of using the latrine, soap usage for hand washing, eating raw vegetables, and habit of soil eating was a determinant factor to soil-transmitted helminthiasis infestation. Therefore, the Preventive chemotherapy program needs to strength, besides its health education activities on Anti-natal care program needs to give focus on latrine utilization, using soap during hand washing, and avoid eating raw vegetable and soil.

\section{Declarations}

\section{Ethics approval and consent to participate}

The study protocol was reviewed and approved by the institutional review board of the University of Gondar, College of Medicine and Health Sciences, Institute of Public Health via the department (Ref No./IPH/1140/2013, date 2/10/2020). Government officials (health and administrative officials) at various levels in the study areas were consulted and permission was obtained from them before data collection. Verbal informed consent was obtained from each study subjects. The issue of confidentiality and privacy was maintained during the training session \& the purpose and benefits of the study were explained to the respondents. Confidentiality of the information was maintained by excluding names and we used identification in the questionnaire and their privacy was kept during the interview by interviewed them alone. Those pregnant women who were positive result was linked to the health center ANC department for treatment. The whole procedure was done according to Helsinki declaration.

\section{Consent to publication}

Not applicable

\section{Availability of data and material}

Data will be available from the corresponding author upon request

\section{Competing Interests}

There is no any competing of interests related with this work

\section{Funding}


This work was supported financially by the University of Gondar. However, the University had no role in study design, data collections and analysis, decision to publish, or preparation of the manuscript.

\section{Acknowledgments}

Our special gratitude goes to the central Gondar zone health department, West Dembia health office and health institutions for their kind cooperation and continuous support. Finally, we would like to extend our gratitude to the study participants, data collectors, and supervisors.

TA conceived the study, developed the tool, coordinated the data collection activity, and carried out the statistical analysis. LD participated in the design of the study, development of the tool, and drafting of the manuscript. MMB and TYA participated in the design of the study and development of the tool, performed the statistical analysis, and drafted the manuscript. All authors read and approved the final manuscript.

\section{References}

1. Farrell SH, Coffeng LE, Truscott JE, Werkman M, Toor J, de Vlas SJ, et al. Investigating the effectiveness of current and modified World Health Organization guidelines for the control of soiltransmitted helminth infections. Clin Infect Dis. 2018;66(suppl_4):253-S9.

2. Organization WH. Soil-transmitted helminthiases: eliminating as public health problem soiltransmitted helminthiases in children: progress report 2001-2010 and strategic plan 2011-2020. World Health Organization; 2012.

3. Bruschi F, Dupouy-Camet J. Helminth infections and their impact on global public health. Springer; 2014.

4. Organization WH. Guideline: preventive chemotherapy to control soil-transmitted helminth infections in at-risk population groups. World Health Organization; 2017.

5. Burssa D, Teshome A, Iverson K, Ahearn O, Ashengo T, Barash D, et al. Safe surgery for all: early lessons from implementing a national government-driven surgical plan in Ethiopia. World journal of surgery. 2017;41(12):3038-45.

6. Mengitsu B, Shafi O, Kebede B, Kebede F, Worku DT, Herero M, et al. Ethiopia and its steps to mobilize resources to achieve 2020 elimination and control goals for neglected tropical diseases: Spider webs joined can tie a lion. International health. 2016;8(suppl_1):i34-52.

7. Samuel F. Status of soil-transmitted helminths infection in Ethiopia. Am J Health Res. 2015;3(3):170-6.

8. Echazú A, Bonanno D, Juarez M, Cajal SP, Heredia V, Caropresi S, et al. Effect of poor access to water and sanitation as risk factors for soil-transmitted helminth infection: selectiveness by the infective route. PLoS Negl Trop Dis. 2015;9(9):e0004111.

9. Mpairwe $H$, Tweyongyere R, Elliott A. Pregnancy and helminth infections. Parasite Immunol. 2014;36(8):328-37. 
10. Cheesbrough M. District laboratory practice in tropical countries, part 2. Cambridge university press; 2005.

11. Montresor A, Mupfasoni D, Mikhailov A, Mwinzi P, Lucianez A, Jamsheed M, et al. The global progress of soil-transmitted helminthiases control in 2020 and World Health Organization targets for 2030. PLoS Negl Trop Dis. 2020;14(8):e0008505.

12. Truscott J, Turner $\mathrm{H}$, Anderson R. What impact will the achievement of the current World Health Organisation targets for anthelmintic treatment coverage in children have on the intensity of soil transmitted helminth infections? Parasites vectors. 2015;8(1):1-12.

13. Tesfaye DJ. Prevalence of intestinal helminthiases and associated factors among pregnant women attending antenatal clinic of Nigist Eleni Mohammed memorial hospital, Hossana, Southern Ethiopia. Open Access Library Journal. 2015;2(07):1.

14. Derso A, Nibret E, Munshea A. Prevalence of intestinal parasitic infections and associated risk factors among pregnant women attending antenatal care center at Felege Hiwot Referral Hospital, northwest Ethiopia. BMC Infect Dis. 2016;16(1):1-7.

15. Mengist $\mathrm{HM}$, Zewdie $\mathrm{O}$, Belew $A$. Intestinal helminthic infection and anemia among pregnant women attending ante-natal care (ANC) in East Wollega, Oromia, Ethiopia. BMC Res Notes. 2017;10(1):1-9.

16. Feleke BE, Jember TH. Prevalence of helminthic infections and determinant factors among pregnant women in Mecha district, Northwest Ethiopia: a cross sectional study. BMC Infect Dis. 2018;18(1):16.

17. Gebrehiwet MG, Medhaniye AA, Alema HB. Prevalence and associated factors of soil transmitted helminthes among pregnant women attending antenatal care in Maytsebri primary hospital, North Ethiopia. BMC Res Notes. 2019;12(1):1-6.

18. Aranzales AFE, Radon K, Froeschl G, Rondón ÁMP, Delius M. Prevalence and risk factors for intestinal parasitic infections in pregnant women residing in three districts of Bogotá, Colombia. BMC Public Health. 2018;18(1):1-15.

19. Yesuf DA, Abdissa LT, Gerbi EA, Tola EK. Prevalence of intestinal parasitic infection and associated factors among pregnant women attending antenatal care at public health facilities in Lalo Kile district, Oromia, Western Ethiopia. BMC Res Notes. 2019;12(1):1-6.

20. Hailu T, Abera B, Mulu W, Kassa S, Genanew A, Amor A. Prevalence and Factors Associated with Intestinal Parasitic Infections among Pregnant Women in West Gojjam Zone, Northwest Ethiopia. Journal of Parasitology Research. 2020;2020.

21. Adewale B, Rahaman O, Aina O, Sulyman MA. Schistosoma mansoni and soil transmitted helminth (STH) infections among pregnant women attending primary health care facilities in Lagos mainland, Nigeria. Journal of Biosciences Medicines. 2018;6(12):64-70.

22. Njeru A, Mutuku F, Muriu S. Status of Soil-transmitted helminthiasis among pregnant women attending antenatal clinic in Kilifi county hospital, Kenya. bioRxiv. 2019:613570.

23. Organization WH. Prevention and control of schistosomiasis and soil-transmitted helminthiasis: World Health Organization/Unicef joint statement. World Health Organization; 2004. 
Figures

\section{The prevalence of Soil-transmitted helminthiasis infection among pregnant women in Ethiopia}

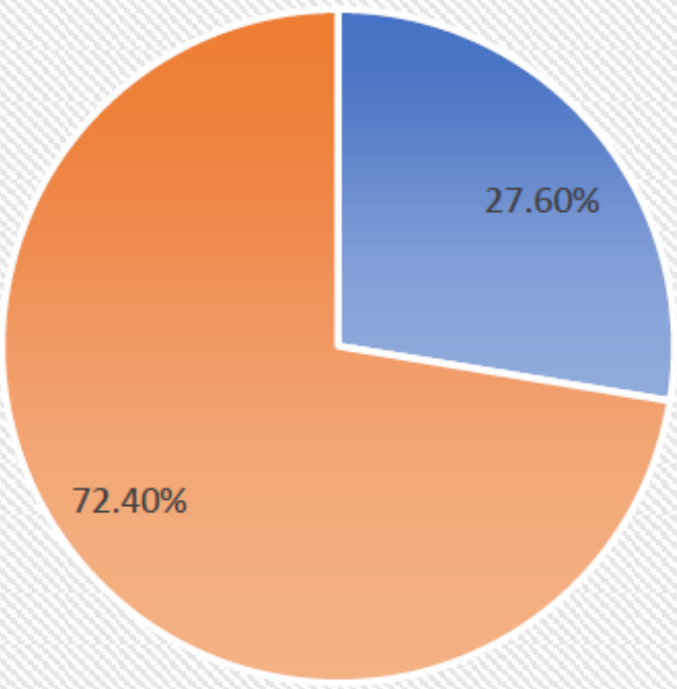

- infected

- not infected

Figure 1

The prevalence of Soil transmitted helminthiasis infection among pregnant women in Ethiopia 